\title{
Anesthesia Management in a Patient with Kabuki Syndrome
}

\author{
Yunus Oktay Atalay ${ }^{1}$, Cengiz Kaya², Yasemin Burcu Ustun², Ali Haydar Sahinoglu² \\ Radiology Department, Outpatient Anesthesia Service, , Faculty of Medicine, Ondokuz Mayis University, Samsun, Turkey ${ }^{1}$ \\ Anesthesiology and Reanimation Department, Faculty of Medicine,Ondokuz Mayis University,Samsun, Turkey²
}

Corresponding author: Yunus Oktay Atalay, MD. Radiology Department, Outpatient Anesthesia Service, , Faculty of Medicine, Ondokuz Mayis University, Samsun, Turkey. Phone:+905056762909. Email: yunus.atalay76@gmail.com

\begin{abstract}
Background and objectives: The aim of this case was to describe the anesthetic approach to a patient with Kabuki syndrome. Case report: A patient with Kabuki syndrome had revision surgery for scoliosis. On physical examination, shown were long palpebral fissures, large, prominent fissures with an eversion of the lateral third of the lower eyelids, large, prominent malformed ears with low implantation, a short nasal septum, micrognathia, thoracolumbar scoliosis, a depressed left shoulder, a low-set occipital hairline and a short neck. The skin was elastic, and joints were lax. The laryngoscopy showed a Grade II Cormack and Lehane view of the larynx. The trachea was intubated easily. The patient was positioned carefully. Vital signs remained stable during surgery. The patient was extubated and transported to the post-anesthetic care unit. Conclusion: Anesthesiologists should be aware of possibly difficult tracheal intubation cardiac lesions, respiratory problems, neurological and musculoskeletal disorders, and a latex allergy when managing anesthesia for a patient with Kabuki syndrome.
\end{abstract}

Key words: Kabuki syndrome, anesthesia, general.

\section{INTRODUCTION}

Kabuki syndrome (KS) is a rare genetic disorder associated with multiple organ abnormalities, unique facial features and mental retardation; it was first described by Niikawa et al., and Kuroki et al. simultaneously in a group of patients in $1981(1,2)$. The facial features of these patients were like the make-up actors in Kabuki dance-drama, traditional Japanese theatre (3). KS has been estimated to occur in 1/32000 in the Japanese population; since 1981, there have also been new reports from other countries (4). The cause of KS is currently unknown. Many cytogenetic abnormalities have been reported, and the most common change is related to chromosome X (1). The diagnosis is considered if the following features, in particular, appear: facial features with long palpebral fissures in which the outer third has an eversion, ptosis, arched eyebrows, prominent eyelashes and ears $(3,5)$. Microcephaly, cardiovascular anomaly, a cleft lip and/or a cleft palate, dental anomalies, malocclusion, microdontia, a small dental arch, joint laxity, scoliosis, urinary tract malformation, hearing loss, seizures and hip dislocation are all other features seen in this syndrome (5). These patients often require operative corrections because of multiple anomalies. The purpose of this case is to review manifestations of KS and to discuss specific anesthetic concerns related to this syndrome. The patient's parent reviewed the case report and gave written permission for publication.

\section{CASE REPORT}

A 9-year oldfemale patient, who weighed $25 \mathrm{~kg}$, was diagnosed with KS and was scheduled for revision surgery for scoliosis. She had received a diagnosis of KS when she was 1 month old. Her past medical history revealed repeated respiratory tract infections (no further infection during the last six months), a delay in motor development and thoraco scoliosis. The patient had no known allergies to medications. She had undergone scoliosis surgery at age 6 and umbilical hernia surgery at age 3. No anesthetic complication was recorded in these surgeries. On physical examination, the following facial features were noted: long palpebral fissures, large, prominent fissures with an eversion of the lateral third of the lower eyelids, large, prominent malformed ears with low implantation, a short nasal septum, micrognathia, thoracolumbar scoliosis, a depressed left shoulder, a low-set occipital hairline and a short neck (Figures 1 and 2). On auscultation, the bilateral lung sounds were clear, a heart murmur was not detected, and the cardiac rhythm and rate were regular. Our patient's echocardiography was normal. The skin was elastic, and the joints showed laxity. Dilatation in the lower pole of the kidney, vesicoureteral reflux and a low capacity bladder were determined in her abdominal ultrasonography. Our patient's chromosomal analysis showed a 46, XX karyotype without any chromosomal abnormality. On the assessment of an airway, we detected micrognathia and a short neck. The Mallampati score was II. We made preparations for apossibly difficult intubation.

She was premedicated with $0.5 \mathrm{mg} / \mathrm{kg}$ oral midazolam. Anesthesia was induced with $8 \%$ sevoflurane in a $50 \%$ nitrous oxide-oxygen mixture. After maintaining a peripheral catheter, rocuronium $0.7 \mathrm{mg} / \mathrm{kg}$ and remifentanil $1 \mu \mathrm{g} / \mathrm{kg}$ were given intravenously. The laryngoscopy showed a Grade II Cormack and Lehane view of the larynx. The trachea was intubated easily with a size 5.0 cuffed orotracheal tube. The patient was positioned carefully because of joint laxity. Anesthesia was maintained with sevoflurane $2 \%$ in a $50 \%$ air-oxygen mixture and an 


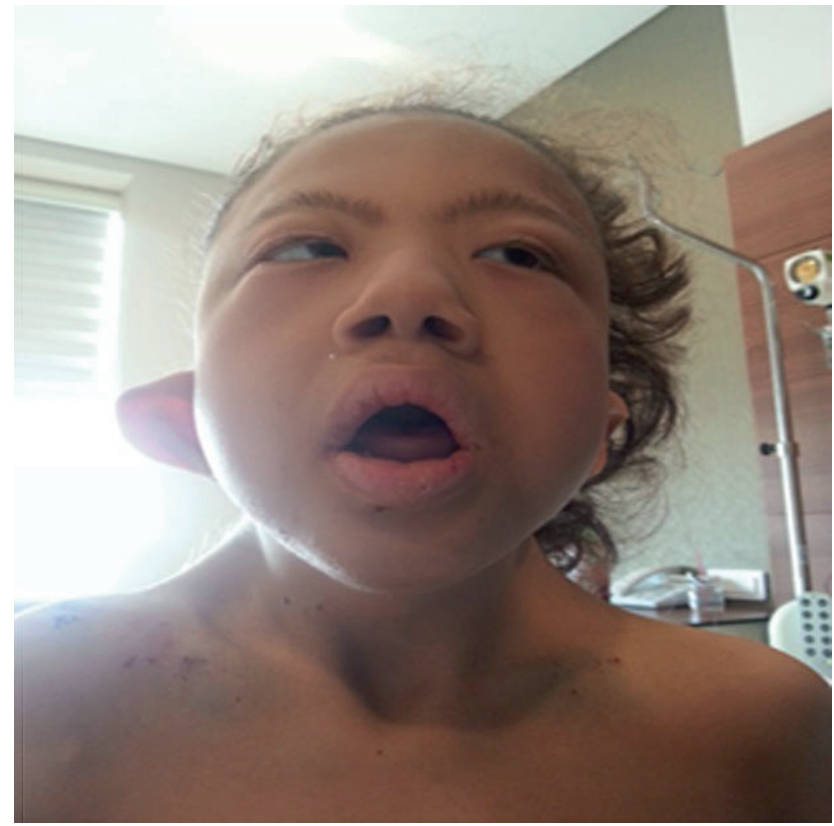

Figure 1. Facial features of Kabuki syndrome. Note the long palpebral fissures with a lower lateral eyelid eversion and a short nasal septum.

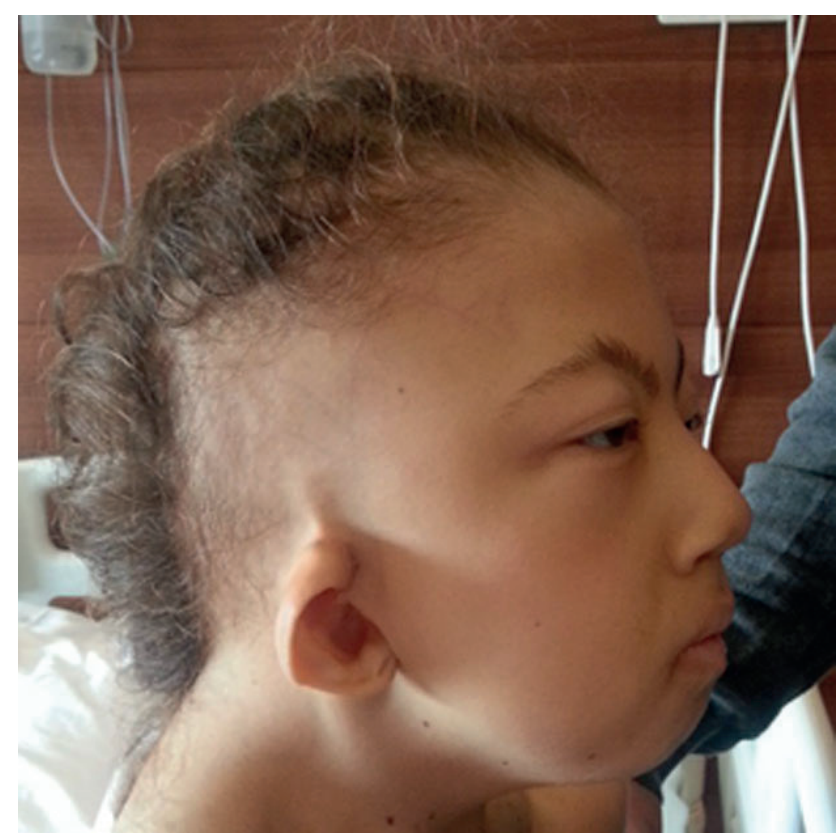

Figure 2. Profile of a patient with Kabuki syndrome. Note the large, prominent malformed ears with low implantation, micrognathia and a short neck.

infusion of remifentanil $0.1-0.2 \mu \mathrm{g} / \mathrm{kg} / \mathrm{min}$. Vital signs remained stable during surgery. The patient was extubated and transported to the post-anesthetic care unit. Morphine $0.05 \mathrm{mg} / \mathrm{kg}$ and paracetamol $15 \mathrm{mg} / \mathrm{kg}$ were given intravenously before extubation for post-operative analgesia. Analgesia was maintained with intravenous, patient-controlled analgesia (continuous infusion rate $5 \mu \mathrm{g} / \mathrm{kg} / \mathrm{hr}$ of morphine and a lockout interval of 8 minutes).

\section{DISCUSSION}

Anesthetic concerns specific to this syndrome relate to possible airway difficulties, congenital heart disease, pulmonary function, joint laxity and a latex allergy. Children with KS may be difficult to intubate as a result of a high arched palate, abnormal dentition, a cleft lip and/or a cleft palate and malocclusion. Approximately one-third of patients have a cleft lip/palate, while two-thirds have a high arched palate (1). Van Haelst et al. reported on two patients: one with local stenosis of the right upper lobe bronchus and the other with severe bronchomalacia and an abnormal right bronchial tree. The anesthesiologist needs to evaluate the airway preoperatively and prepare for possible difficulty (2).

The patients with KS may have some congenital heart defects, and the prevalence of these defects in this population has been estimated to be from $40 \%$ to $50 \%$ (1). Cardiac abnormalities include atrial and ventricular septal defects, coarctation of the aorta, a bicuspid aortic valve, mitral valve prolapse and hypertrophic cardiomyopathy (3). The selection of anesthetics is critical, especially for a patient who has poor ventricular function. Preoperative assessment of the patient's cardiac lesions and functions will be useful. Patients with KS usually have normal pulmonary function, but recurrent pneumonia can be seen as being related to immune dysfunction. Obstructive sleep apnea is common, and the patient may require a tonsillectomy (1). Genitourinary and gastrointestinal anomalies, including anal atresia, diaphragmatic hernia and eventrations, ptosis and strabismus, increase susceptibility to infections, autoimmune disorders, endocrinological abnormalities (like thelarche in females); feeding problems, hearing loss, seizures and hypotonia are other abnormalities associated with $\mathrm{KS}(1,4,5)$. Muscular hypotonia is a common feature; there may be concern about a neuromuscular blockade and malignant hyperthermia, but the muscle biopsies have been normal. A larger dose of nondepolarizing muscle relaxant may be required because of the concomitant anti-convulsant therapy (6). Joint laxity and dislocations are common in these patients. Hips, patellae and shoulders are especially affected, so caution must be taken while positioning the patient (1). Teixeira et al. reported a case of a latex allergy in a patient with KS. The patient had a history of two previous reactions in different surgeries, so questions about a possible allergy to latex when taking medical history would help alert the anesthesiologist(7).

\section{CONCLUSION}

Anesthesiologists should be aware of a possibly difficult tracheal intubation, cardiac lesions, respiratory problems, neurological and musculoskeletal disorders and a latex allergy when dealing with the anesthetic management of a patient with Kabuki syndrome.

\section{CONFLICT OF INTEREST: NONE DECLARED}

\section{REFERENCES}

1. Adam MP, Hudgins L. Kabuki syndrome: a review. Clin Genet. 2005; 67(3): 209-219.

2. Casado AI, Ruiz J, Oro J, Martinez C, Fernandez I, Oliva P. Anaesthetic management in a case of Kabuki syndrome. Eur J Anaesthesiol. 2004; 21(2):162-163.

3. Jones KL. Smith's Recognizable Patterns of Human Malformation. 6th ed. Philadelphia: WB Saunders. 2005: 118-119..

4. Matsumoto N, Niikawa N. Kabuki make-up syndrome: a review. Am J Med Genet C Semin Med Genet. 2003; 117(1):57-65.

5. Matsumura M, Yamada R, Kitani Y, Nishi T, Yamamoto H, Oahama $\mathrm{Y}$, et al. Anorectal anomalies associated with Kabuki make-up syndrome. J Pediatr Surg. 1992; 27(12): 1600-1602.

6. Johnson G, Mayhew JF. Anesthesia for a child with Kabuki Syndrome. Paediatr Anaesth. 2007; 17(9): 900-901.

7. Teixeira VC, Neves MA, de Castro RA. Latex allergy in a patient with Kabuki syndrome. Case report. Rev Bras Anestesiol. 2010; 60(5): 544-550. 